\title{
Higher constitutive IL15R $\alpha$ expression and lower IL-15 response threshold in coeliac disease patients
}

D. Bernardo, ${ }^{\star}$ J. A. Garrote, ${ }^{\star, \dagger}$

Y. Allegretti, ${ }^{\star}$ A. León, ${ }^{\star}$ E. Gómez, ${ }^{\star}$

J. F. Bermejo-Martin, ${ }^{\star}$ C. Calvo, ${ }^{\star}$,

S. Riestra, ${ }^{9}$ L. Fernández-Salazar, ${ }^{\dagger \dagger}$

A. Blanco-Quirós, ${ }^{\star}$ F. Chirdo ${ }^{\star}$ and

E. Arranz ${ }^{*}$

${ }^{*}$ Mucosal Immunology Laboratory, Instituto de Biología y Genética Molecular (IBGM),

Universidad de Valladolid-CSIC, ${ }^{\dagger}$ Research Unit,

${ }^{\S}$ Paediatrics Service, and ${ }^{\dagger \dagger}$ Gastroenterology

Service, Hospital Clínico Universitario,

Valladolid, Spain, ${ }^{\ddagger}$ Laboratorio de Investigación en el Sistema Inmune (LISIN) Facultad de Ciencias Exactas, Universidad Nacional de La Plata, La Plata, Argentina, and 'Digestive Diseases Service, Hospital Universitario Central de Asturias, Oviedo, Spain

Accepted for publication 3 July 2008 Correspondence: E. Arranz, Mucosal Immunology Laboratory, Department of Paediatrics and Immunology, Universidad de Valladolid, C/Ramón y Cajal, 7. 47005. Valladolid, Spain. E-mail: earranz@med.uva.es

\section{Summary}

The IL-15 triggering effect of gliadin is not exclusive to coeliac disease (CD) patients, whereas the secondary response is $\mathrm{CD}$ specific. We have studied the expression of the IL-15 receptor, and the IL-15 response upon stimulation, in non-CD and $\mathrm{CD}$ patients, and the possible existence of a lower immunological threshold in the latter. Forty-two CD patients ( 20 on a gluten-containing diet, GCD, and 22 on gluten-free diet, GFD) and 24 non-CD healthy individuals were studied. IL15R $\alpha$ mRNA expression, and tissue characterization, were assayed in the duodenum. Biopsies from six CD patients on GFD and 10 non-CD individuals were studied in vitro using organ culture in basal conditions, as well as after IL-15 stimulation discarding basal IL-15 production. Secretion of immune mediators was measured in the culture supernatants. IL15R $\alpha$ mRNA expression was increased in CD patients, as compared with non-CD controls (on GFD $P=0.0334$, on GCD $P=0.0062$, respectively), and confirmed also by immunofluorescence. No differences were found between CD patients on GFD and on GCD. After in vitro IL-15 stimulation, IL15R $\alpha$ expression was only triggered in non-CD controls $(P=0.0313)$, though it remained increased in CD patients. Moreover, IL-15 induced a more intense immunological response in $\mathrm{CD}$ patients after triggering the production of both nitrites and $\operatorname{IFN} \gamma(P=0.0313, P=0.0313$, respectively). Gliadin-induced IL15 has a lower response threshold in CD patients, leading to the production of other immune mediators and the development of the intestinal lesion, and thus magnifying its effects within the $\mathrm{CD}$ intestine.

Keywords: Coeliac disease, IL-15, IL15R, immune threshold

\section{Introduction}

Coeliac disease (CD) is a small intestinal hypersensitivity to wheat and other gluten-containing cereals (rye, barley and probably oats) occurring in genetically predisposed individuals $[1,2]$. This immune-mediated enteropathy is characterized by villous atrophy, crypt hyperplasia and increased infiltration by intraepithelial lymphocytes [3]. Some gluten peptides reach to the lamina propria, where some of them are deaminated by the enzyme tissue transglutaminase (TG2) [4] and presented on a HLA-DQ2 (or DQ8) context by dendritic cells $[5,6]$, with the subsequent induction of a pro-inflammatory response, mainly characterized by IFN $\gamma$ production. The current treatment is a life-long strict gluten-free diet (GFD), which results in a complete remission of symptoms and mucosal histology [1,2].
Nowadays, the most accepted model for explaining the immunopathogenesis of CD is the two-signal model [7]. Thus, innate immunity plays a key role, through a DQ2independent mechanism, in the development of $\mathrm{CD}[8,9]$. In this context, gliadin toxic peptides (i.e. the 19-mer) trigger an innate immune response [10], mainly characterized by the production of IL-15 by epithelial cells [11]. The result is the disruption of the epithelial barrier, both by directly increasing the permeability throughout the tight-junctions $[12,13]$, and by inducing enterocyte apoptosis after intraepithelial lymphocyte reprogramming into NK-like cells [14-17]. As a consequence, immuno-adaptive peptides, like the 33-mer, can now reach to the lamina propria, where they are presented by dendritic cells to gluten-specific T cells [18], acting as a bridge between the innate and the adaptive immunity [19]. 
This innate response has been proposed to be CD specific [10], but no differential factors controlling this response have been described so far. Moreover, by using a biopsy culture model, we have previously shown that the IL-15 triggering effect of gliadin occurs in both $\mathrm{CD}$ patients and non-CD healthy controls, though the adaptive immune mediators were only elicited by CD patients [20].

As the first gluten-induced (innate) signal is observed in both $\mathrm{CD}$ and non-CD individuals, whereas the second (adaptive) signal appears specifically in CD patients, and considering that duodenal biopsies of CD patients showed an increased expression of IL15R $\alpha$ [11], it is tempting to speculate that the higher expression of this receptor has a biological role in the immunopathogenesis of CD. Thus, the gliadin-induced IL-15 production would lead to major changes in CD patients, but it would be not enough to reach the threshold response in non-CD patients.

To address this question, basal expression of IL15R $\alpha$ mRNA was determined in CD patients with active disease and on a GFD, and in a group of healthy controls. Moreover, given the higher expression of IL15R $\alpha$ in CD patients, biopsies from both non-CD healthy controls and CD patients on GFD were studied in vitro using organ culture in basal conditions, and after IL-15 stimulation, in order to evaluate the production of secondary immune mediators.

\section{Materials and methods}

\section{Study subjects}

We studied a total of $42 \mathrm{CD}$ patients (mean age 21.24 years, range 2-68 years, 30.95\% males); 20 were untreated (mean age $6 \cdot 80$ years, range $2-38,30 \cdot 00 \%$ males) and 22 treated on a GFD (mean age 34.36 years, range $7-68$ years, $31 \cdot 82 \%$ males). A group of 24 non-CD healthy individuals were included in our study as controls (mean age 53.04 years, range $16-81$ years, $20 \cdot 83 \%$ males). They were referred to the Gastroenterology Clinics due to other intestinal pathologies, which were later ruled out, and no mucosal changes were found in the duodenum. The final diagnosis was non-CD in all cases. At the time of diagnosis, all CD patients had compatible symptoms of the disease, positive serology (IgA antiendomysial or antitransglutaminase antibodies), genetics (HLA-DQ2/8), and mucosal changes in the duodenal biopsy. At the time of sample collection, active CD patients had positive serology and mucosal changes, whereas CD patients on GFD had mucosal recovery (Marsh 0-1) and negative serology for at least 1 year. The recruited patients attended the Adult Gastroenterology Clinics at the 'Hospital Clínico Universitario' in Valladolid, and the 'Hospital Valle del Nalón' in Asturies, as part of the routine diagnostic procedures. Informed consent was obtained from patients, and the study protocol was approved by the Ethics Committee of both 'Hospital Clínico Universitario' and the Faculty of Medicine, University of Valladolid.

\section{Biopsy and specimen preparation}

A total of 66 biopsies, 24 from healthy controls and 42 from CD patients (20 on GFD and 22 on a gluten-containing diet) were collected. They were immediately submerged in $0.5 \mathrm{ml}$ of RNALater ${ }^{\circledR}$ solution (Ambion Inc., Austin, Texas, USA) and stored at $-20^{\circ} \mathrm{C}$. Both total RNA and proteins were isolated from each biopsy using the TRIZOL ${ }^{\circledR}$ reagent according to the protocol provided by the manufacturer. Reverse transcription was carried out by using the SuperScript ${ }^{\circledR}$ FirstStrand Synthesis System for reverse Transcriptase (RT)-PCR Kit (Invitrogen, Life Technologies, Carlsbad, CA, USA) using OligodT primers.

\section{Organ culture of duodenal mucosa}

Intestinal biopsies from $10 \mathrm{CD}$ patients on GFD (mean age 43.64 years, range $23-68,18 \cdot 18 \%$ males) and 23 non-CD healthy controls (mean age 53.04 years, range $16-81,20 \cdot 83 \%$ males) were cultured in vitro as previously described [21]. Briefly, all biopsies were collected in ice-chilled PBS (Cambrex Iberia Products, Barcelona, Spain) containing $0 \cdot 1 \%$ Gentamicine (Cambrex) and cultured within $1 \mathrm{~h}$ in RPMI 1640 (Cambrex) supplemented with 10\% heatinactivated FBS (Cambrex), penicillin $(100 \mathrm{U} / \mathrm{mL})$, streptomycin $(100 \mu \mathrm{g} / \mathrm{ml})($ Cambrex $)$ and fungizone $(0 \cdot 25 \mu \mathrm{g} / \mathrm{ml})$ (Cambrex). Seven CD patients on a GFD and 10 non-CD healthy controls were also studied after IL-15 stimulation with $50 \mathrm{ng} / \mathrm{ml}$ (Pierce Biotechnology Inc., Rockford, IL, USA) (patients on GFD, mean age $47 \cdot 71$ years, range 25-68, $0 \%$ males; non-CD healthy controls mean age 53.6 years, range $23-66,10 \%$ males) (Table 1 ). In these cases, each sample cultured in basal conditions constituted an internal control. Tissue culture was carried out by immersion in culture dishes placed in a cell incubator with $5 \% \mathrm{CO}_{2}$ at $37^{\circ} \mathrm{C}$. After 3 hours, biopsy specimens were washed up in PBS containing $0 \cdot 1 \%$ Gentamicine (Cambrex) and later cultured for another $21 \mathrm{~h}$ in new clean culture medium to determine whether an innate stimulus is followed by a secondary response. After $24 \mathrm{~h}$ ( $3 \mathrm{~h}$ with stimulus $+21 \mathrm{~h}$ of basal medium), tissue was embedded in RNAlater (Ambion) and snap-frozen until protein and RNA isolation extraction were carried out as previously described.

\section{Western blot analysis}

Eight $\mu \mathrm{g}$ of protein isolated from whole biopsy explants were added per well. They were separated by using a $15 \%$ acrilamide/bisacrilamide (37.5:1) gel in a mini-Protean II (BioRad Laboratories Inc., Hercules, CA, USA), and later transferred onto PVDF membranes of 0.45 Micron (Pierce Biotechnology Inc.). Membranes were incubated with primary specific antibodies to human IL-15 (mouse monoclonal MAB247, R\&D, Minneapolis, USA) at a final dilution 
Table 1. Coeliac disease (CD) patients treated on a gluten-free diet (GFD) (a) and non-coeliac patients (b) biopsy cultured in basal medium and IL-15 challenged. All treated CD patients had negative serology and no duodenal alterations.

\begin{tabular}{|c|c|c|c|c|c|c|c|}
\hline (a) Case & Age & Gender & Months on a GFD & Duodenal mucosa & Serology & HLA-DQ & Basal IL-15 \\
\hline GFD-CD 1 & 44 & Female & 18 & Marsh 1 & Negative & $\mathrm{DQ} 2+$ & - \\
\hline GFD-CD 2 & 25 & Female & 54 & Marsh 1 & Negative & $\mathrm{DQ} 2+$ & - \\
\hline GFD-CD 3 & 68 & Female & 123 & Marsh 0 & Negative & $\mathrm{DQ} 2+$ & + \\
\hline GFD-CD 4 & 48 & Female & 18 & Marsh 1 & Negative & $\mathrm{DQ} 2+$ & - \\
\hline GFD-CD 5 & 44 & Female & 12 & Marsh 1 & Negative & $\mathrm{DQ} 2+$ & - \\
\hline GFD-CD 6 & 66 & Female & 14 & Marsh 1 & Negative & $\mathrm{DQ} 2+$ & - \\
\hline GFD-CD 7 & 39 & Female & 13 & Marsh 1 & Negative & $\mathrm{DQ} 2+$ & - \\
\hline (b) Case & & Age & Gender & \multicolumn{3}{|c|}{ Diagnosis } & Basal IL-15 \\
\hline Non-CD 1 & & 33 & Female & \multicolumn{3}{|c|}{ Functional dyspepsia } & - \\
\hline Non-CD 2 & & 58 & Female & \multicolumn{3}{|c|}{ Functional dyspepsia } & - \\
\hline Non-CD 3 & & 53 & Female & \multicolumn{3}{|c|}{ Hiatus hernia } & - \\
\hline Non-CD 4 & & 60 & Female & \multicolumn{3}{|c|}{ Chronic gastritis } & - \\
\hline Non-CD 5 & & 53 & Female & \multicolumn{3}{|c|}{ Functional dyspepsia } & - \\
\hline Non-CD 6 & & 60 & Male & \multicolumn{3}{|c|}{ Functional dyspepsia } & - \\
\hline Non-CD 7 & & 73 & Female & \multicolumn{3}{|c|}{ Functional dyspepsia } & - \\
\hline Non-CD 8 & & 69 & Female & \multicolumn{3}{|c|}{ Functional dyspepsia } & - \\
\hline Non-CD 9 & & 56 & Female & \multicolumn{3}{|c|}{ Chronic gastritis } & - \\
\hline Non-CD 10 & & 23 & Female & \multicolumn{3}{|c|}{ Functional dyspepsia } & - \\
\hline
\end{tabular}

of $1 / 400$, performing a second incubation with antibodies to mouse IgG labelled with horseradish peroxidase (Amersham Biosciences Europe, Freiburg, Germany). Chemiluminiscent substrate Lumigen PS-3 (Amersham) and autoradiography film Hyperfilm ECL (Amersham) were used for developing. Recombinant human IL-15 (Peprotech, London, UK) was used as a positive control.

\section{Immunofluorescence studies}

Duodenal biopsies from three healthy controls and three CD patients on a gluten-containing diet were also fixed in Bowin's medium and included in paraffin. Sections were rehydrated and treated with antigen retrival solution (Dako, Glostrup, Denmark). After two washes in PBS-Tween20 $0 \cdot 1 \%$, samples were sequentially incubated with $50 \mu \mathrm{g} / \mathrm{ml}$ of goat anti-IL15R $\alpha(\mathrm{R} \& \mathrm{D})$, and donkey anti-goat IgG-Cy5 1:200 (Jackson ImmunoResearch Laboratories, West Grove, PA, USA). Images were taken in a LSM 510 meta Zeiss confocal microscopy using the LSM 5 v 3.2 software.

\section{Quantitative polymerase chain reaction}

mRNA levels of IL15R $\alpha$, iNOS, IFN $\gamma$, TNF $\alpha$ and $\beta$-actin (as a house keeping gene) were measured by real-time PCR by using a LightCycler ${ }^{\circledR}$ instrument (Roche Applied Science, Mannheim, Germany). As there are at least eight different isoforms for IL15R $\alpha$ and some of them are secreted [22], IL15R $\alpha$ reverse primer was localized between exons 6 and 7, amplifying only non-secreted isoforms. Forward primer was localized in exon 3, in the linker between the cytokinebinding domain and the Pro/Thr rich domain [22].
Reactions were performed using the FastStart SYBR Green MasterMix (Roche) with thermolabile Uracil DNA Glycosylase (UDG) (Roche) to prevent carry-over contamination. Cytokine primer sets and PCR conditions are described in Table 2. mRNA levels are expressed as the ratio molecule/ $\beta$ actin in arbitrary units. Molecules showing not detectable levels were given an arbitrary unit of $0 \cdot 1$.

\section{Effector molecules on culture supernantants}

According to the Griess Reagent kit for nitrite determination (Molecular Probes, detection limit $1 \mu \mathrm{M}$ ), 3-h biopsy culture supernatants were assayed following the manufacturer's instructions. Final supernatants (after $24 \mathrm{~h}$ of culture) were also analysed by using a multiplex assay (Biorad, Hercules, CA, USA) on a Luminex TM platform (Biorad, Austin, TX, USA), following the manufacturer's instructions, for the concentration of interferon $\gamma$ (IFN- $\gamma$ ) (detection limit (D.L.) $0.542 \mathrm{pg} / \mathrm{ml}$ ), tumour necrosis factor $\alpha$ (TNF- $\alpha$ ) (D.L. $0 \cdot 356 \mathrm{pg} / \mathrm{ml}$ ), interleukin (IL)-12p70 (D.L. 0.927 pg/ml) and IL-2 (D.L. $4 \cdot 422 \mathrm{mg} / \mathrm{ml}$ ). Values below detection levels were reported as being equal to the level of detection.

\section{Statistical analysis}

Non-parametric statistical analyses of the mRNA, nitrites or protein expression levels among groups (non-CD, GFD-CD or active $\mathrm{CD}$ ) were performed using the Kruskal-Wallis oneway analysis of variance test and the Mann-Whitney $U$-test. The Wilcoxon matched paired test was used to compare different culture conditions from the same patient. The level of significance was fixed at $P<0 \cdot 05$. 
Table 2. Cytokine primer sets, PCR products (bp, base pairs), temperature of annealing, and source of the primers used for quantitative PCR.

\begin{tabular}{|c|c|c|c|c|}
\hline Molecule & Primers sequence & $\mathrm{bp}$ & Annealing & Primers source reference \\
\hline$\beta$-actin & $\begin{array}{l}\text { fw: } 5^{\prime}-\text { ATG GGT CAG AAG GAT TCC TAT GTG - 3' } \\
\text { rv: 5' - CTT CAT GAG GTA GTC AGT CAG GTC - } 3^{\prime}\end{array}$ & 359 & 60 & 23 \\
\hline IL15R $\alpha$ & $\begin{array}{l}\text { fw: } 5^{\prime} \text { - GCC AGC GCC ACC CTC CAC AGT AA - 3' } \\
\text { rv: 5' - GCC AGC GGG GGA GTT TGC CTT GAC - } 3^{\prime}\end{array}$ & 402 & 70 & 24 \\
\hline iNOS & $\begin{array}{l}\text { fw: } 5^{\prime}-\text { TCT GCA GAC ACG TGC GTT ACT }-3^{\prime} \\
\text { rv: } 5^{\prime}-\text { ATG CAC AGC TGA GCA TTC CA - } 3^{\prime}\end{array}$ & 115 & 56 & 25 \\
\hline IFN $\gamma$ & $\begin{array}{l}\text { fw: } 5^{\prime}-\text { TGG AAA GAG GAG AGT GAC AG - } 3^{\prime} \\
\text { rv: 5' - ATT CAT GTC TTC CTT GAT GG - } 3^{\prime}\end{array}$ & 129 & 60 & 26 \\
\hline TNF $\alpha$ & $\begin{array}{l}\text { fw: 5' - TCA GAT CAT CTT CTC GAA CC - 3' } \\
\text { rv: } 5^{\prime} \text { - CAG ATA GAT GGG CTC ATA CC }-3^{\prime}\end{array}$ & 361 & 60 & 27 \\
\hline
\end{tabular}

\section{Results}

\section{Higher IL15R $\alpha$ mRNA expression in CD patients}

IL15R $\alpha$ mRNA expression was studied in non-cultured biopsy explants from CD patients, both on GFD and on a gluten-containing diet, and non-CD healthy controls. Neither gender- nor age-related effects were observed in any case (data not shown). Figure 1 shows that the expression of IL15R $\alpha$ mRNA is higher in CD patients, with independence of gluten ingestion (CD patients on GFD, median 4.320 U; $\mathrm{CD}$ patients on a gluten-containing diet, median 7.921 U), when compared with non-CD controls (median $0.795 \mathrm{U}$, $P=0.0334$ and $P=0.0062$, respectively). No statistical differences were found between CD patients on GFD and on a gluten-containing diet (active), which discards higher values because of inflammation, and points to a constitutive and/or primary condition in CD patients. In a similar way, no cor- relation was found between the degree of histological lesion in a gluten-containing diet patient or the months on a GFD in treated patients with the expression of IL15R $\alpha$. Finally, it is noteworthy that the IL15R $\alpha$ mRNA expression shows a bi-modal distribution, as can be observed in the non-CD group. Therefore, the few cases in the latter group with detectable levels of IL15R $\alpha$ were in the range of CD patients.

\section{Tissue IL15R $\alpha$ expression in CD patients}

Confocal microscopy analysis confirmed previous results of higher IL15R $\alpha$ expression in CD patients. Thus, a large number of IL15R $\alpha^{+}$cells were identified within the epithelia, lamina propria and crypt area, in sections from CD patients on a gluten-containing diet (Fig. 2a). On the contrary, rare positive cells, located within the lamina propria, were observed in normal tissue (Fig. 2b). IL15R $\alpha$ was found mainly associated with the nuclear membrane, as previously
Fig. 1. Intestinal expression of IL15R $\alpha$ mRNA in arbitrary units (U) in non-cultured biopsy explants from non-CD healthy controls (C, $n=22)$, and CD patients, both on gluten-free diet (GFD, $n=20)$ and on a diet containing gluten (active, $n=19$ ). Only isoforms non-secreted and carrying exon 3 are detected. Statistically significant differences are shown $(P<0.05$ Krustal-Wallis one-way analysis of variance test and two-tailed Mann-Whitney $U$-test). Horizontal bars indicate median values. IQR: interquartile range.

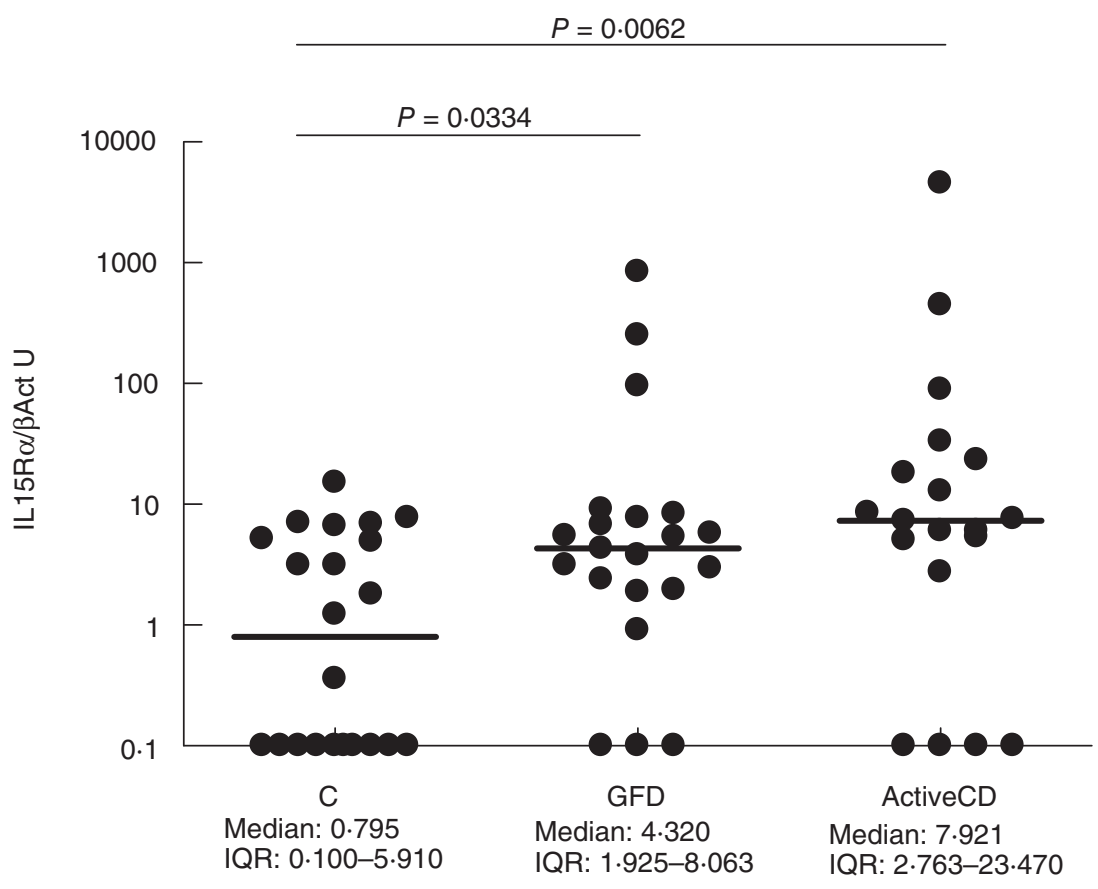


(a)

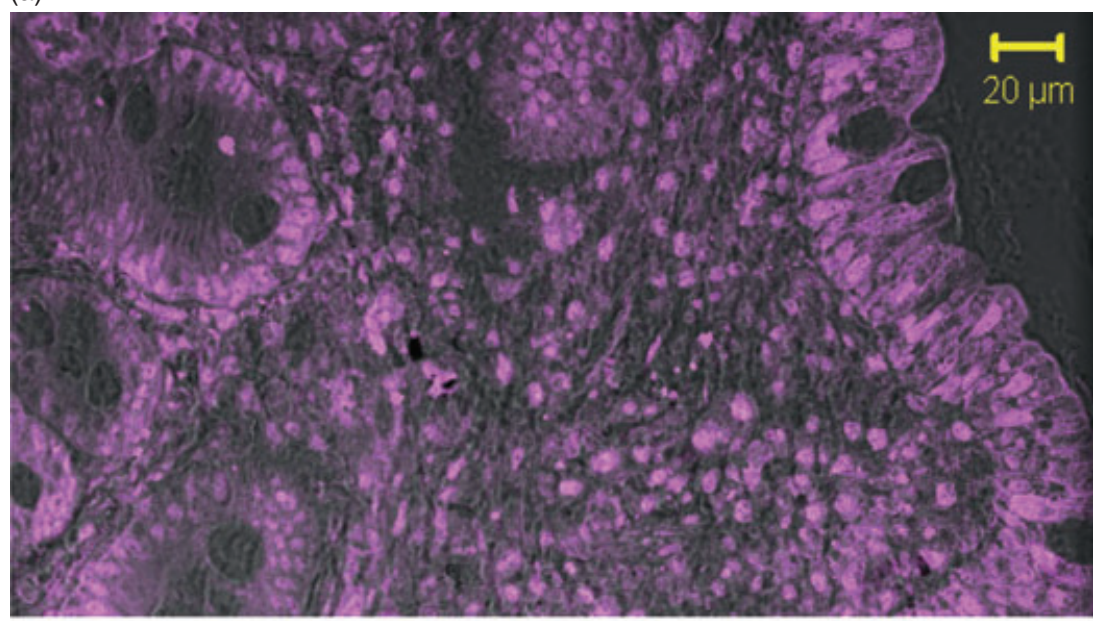

(b)

Fig. 2. Comparison of IL $15 R \alpha$ intestinal expression by confocal immunofluorescence: (a) coeliac patient and (b) non-coeliac healthy control. IL15R $\alpha$ was mainly found associated with the nuclear membrane as previously reported [28].

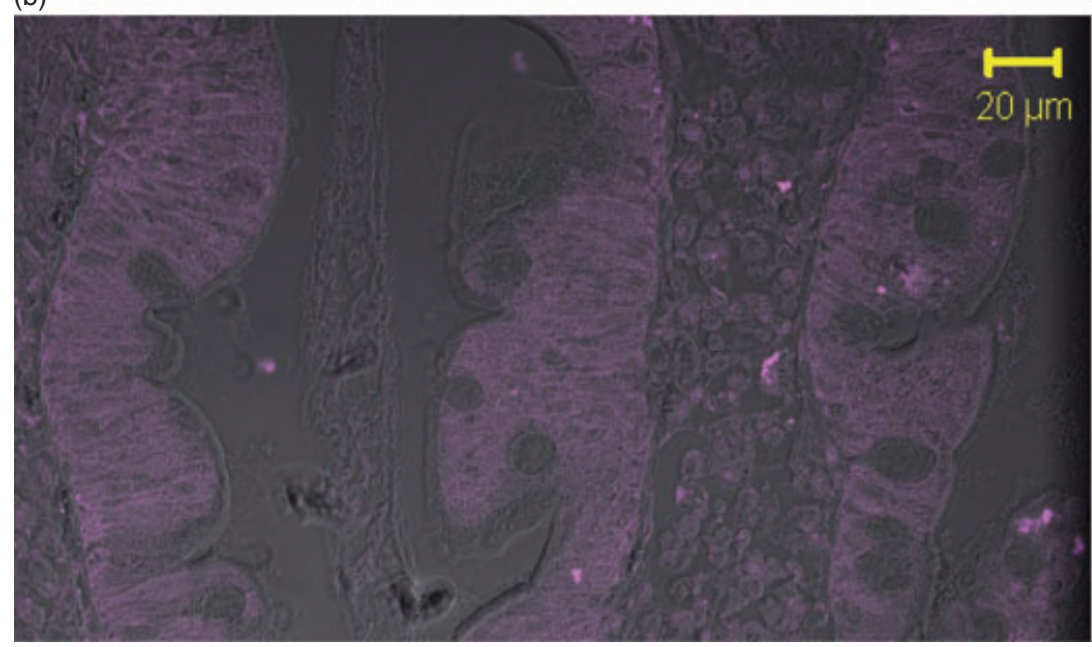

reported [28]. As only those isoforms lacking exon 2 (unable to bind IL-15) do not co-localize with the nuclear membrane, our results confirm the existence of differences in a biologically active IL15R $\alpha$ between CD and non-CD individuals. Based on the morphology and location of IL15R $\alpha+$ cells, it can be concluded that in the intestinal mucosal of CD patients, these cells belong to different lineages.

\section{IL15R $\alpha$ mRNA expression is triggered by in vitro IL-15 stimulation of biopsy explants from non-CD patients}

Next, we performed functional studies addressing the modulation of IL15R $\alpha$ by IL-15 stimulation of biopsies from both
non-CD (10 patients) and CD patients on GFD (7 patients). After organ culture in basal conditions, whole protein biopsy explants were tested for IL-15 by Western blot. Basal IL-15 production was only found in one CD patient, who was discarded from the study in order to correctly measure the IL-15 response to an exogenous stimulus (Fig. 3). Therefore, IL-15 stimulation was performed in 6 GFD-CD and 10 non-CD patients. After $3 \mathrm{~h}$ of IL-15 stimulation and 21 extra $\mathrm{h}$ of culture in basal conditions, IL15R $\alpha$ expression was only triggered in non-CD patients (Fig. 4a) (basal conditions median, 0.100 U; IL-15 stimulation median, 1.204 U; $P=0 \cdot 0313$ ), but not in CD patients (basal conditions median, 6.350 U; IL-15 stimulation median, 10.400 U; $P=$ n.s.).

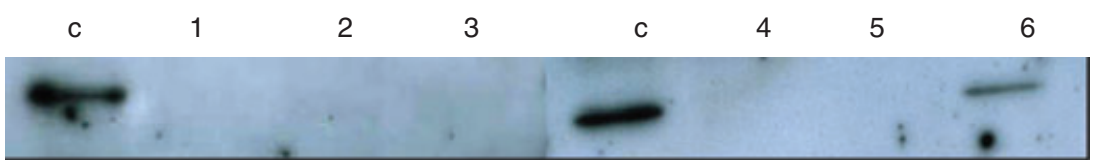

Fig. 3. Representative Western blot analysis using whole protein biopsy explants after 24 h of basal culture in non-coeliac controls (lanes $1-3$ ) and treated coeliac disease patients (lanes 4-6). C: human recombinant IL-15 lane. No detectable levels of IL-15 were found in the non-CD patients, while only one out of seven CD patients on GFD was positive for this cytokine (lane 6). 
(a)

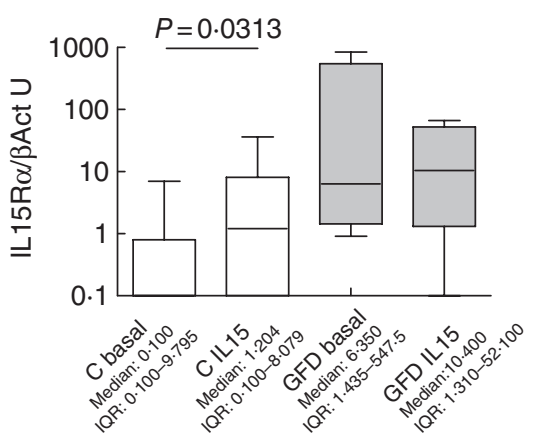

(d)

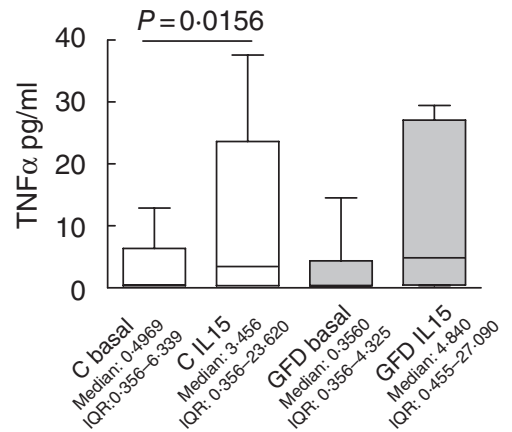

(b)

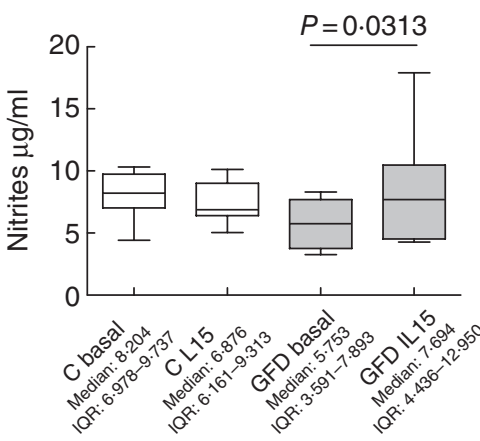

(e)

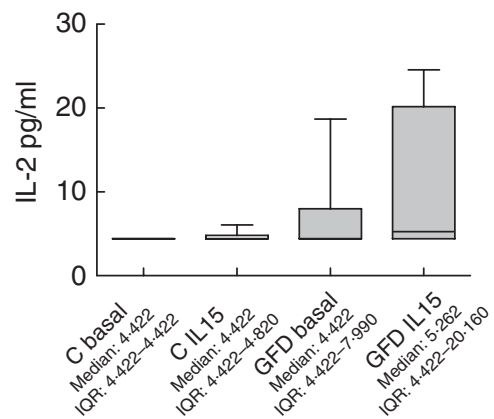

(c)

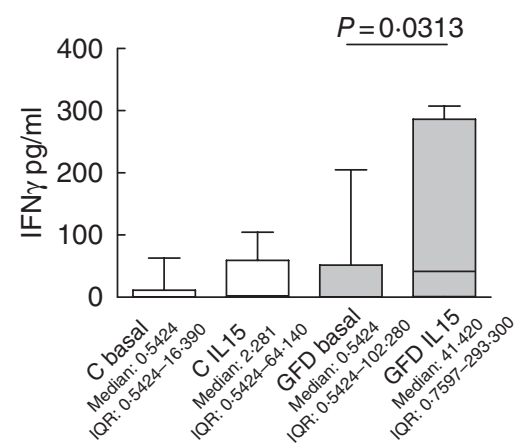

(f)

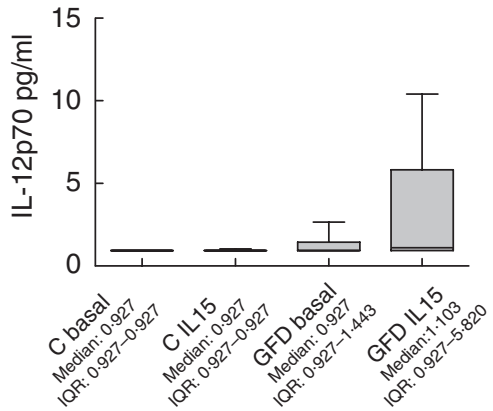

Fig. 4. Organ culture of duodenal biopsy samples: in vitro expression of IL15R $\alpha$ mRNA after $24 \mathrm{~h}$ (a); and secretion of nitrites (b), after $3 \mathrm{~h}$, and IFN $\gamma(\mathrm{c})$, TNF $\alpha$ (d), IL-2 (e) and IL-12p70 (f), after $24 \mathrm{~h}$, from non-CD healthy controls (C, $n=10)$ and coeliac disease patients on GFD ( $n=6)$, in arbitrary units $(\mathrm{U})$, both basal culture conditions and after IL-15 stimulation ( $3 \mathrm{~h}$ of stimulation $+21 \mathrm{~h}$ in basal conditions). Statistically significant differences are shown $(P<0 \cdot 05$, Wilcoxon matched paired test $)$. Horizontal bars indicate median and whiskers maximum and minimum values. IQR: interquartile range.

\section{Increased immunological response to in vitro IL-15 stimulation in treated CD patients}

Organ cultures of biopsy explants were also performed to investigate whether the higher expression of IL15R $\alpha$ observed in CD patients could be involved in a higher IL-15 response in these patients. Interestingly, after only $3 \mathrm{~h}$ of stimulation, innate mediators (i.e. nitrites) were already increased in CD cultures (basal conditions median, $5 \cdot 753 \mu \mathrm{g} /$ ml; IL-15 stimulation median, $7 \cdot 694 \mu \mathrm{g} / \mathrm{ml} ; P=0 \cdot 0313$ ), but not in non-CD (basal conditions median, 8.204 $\mu \mathrm{g} / \mathrm{ml}$; IL-15 stimulation median, $6 \cdot 876 \mu \mathrm{g} / \mathrm{ml}$ ) (Fig. $4 \mathrm{~b}$ ). Moreover, at the end of the culture $(24 \mathrm{~h})$, the pro-inflammatory mediator IFN $\gamma$ was also triggered only in CD patients after IL-15 stimulation (basal conditions median, $0.5424 \mathrm{pg} / \mathrm{ml}$; IL-15 stimulation median, $41.420 \mathrm{pg} / \mathrm{ml} ; P=0.0313$ ) (Fig. 4c), but no changes were observed in non-CD patients (basal conditions median, $0 \cdot 5424 \mathrm{pg} / \mathrm{ml}$; IL-15 stimulation median, $2 \cdot 281 \mathrm{pg} / \mathrm{ml})$. On the other hand, TNF $\alpha$ was reported to be increased, although borderline significant in $\mathrm{CD}$, in both non-CD (basal conditions median, $0.4969 \mathrm{pg} / \mathrm{ml}$; IL-15 stimulation median, $3.456 \mathrm{pg} / \mathrm{ml} ; P=0.0156)$ and $\mathrm{CD}$ patients (basal conditions median, $0.2560 \mathrm{pg} / \mathrm{ml}$; IL-15 stimulation median, $4.840 \mathrm{pg} / \mathrm{ml} ; \quad P=0.0625$ ) (Fig. 4d). Finally, neither IL-2 (non-CD patients, basal conditions median, $4.422 \mathrm{pg} / \mathrm{ml}$, IL-15 stimulation median, $4.422 \mathrm{pg} /$ $\mathrm{ml}$; CD patients, basal conditions median, $4.422 \mathrm{pg} / \mathrm{ml}$, IL-15 stimulation median, 5.262) (Fig. 4e) nor IL-12p70 levels (non-CD patients basal conditions median, $0.927 \mathrm{pg} /$ ml, IL-15 stimulation median, $0.927 \mathrm{pg} / \mathrm{ml}$; CD patients, basal conditions median, $0.927 \mathrm{pg} / \mathrm{ml}$, IL-15 stimulation median, 1.103) (Fig. 4f) were modified after stimulation with IL-15.

\section{Increased basal expression in vitro of pro-inflammatory mediators in treated CD patients}

After discarding differences in the secretion of inflammatory mediators (nitrites, IFN $\gamma$, TNF $\alpha$, IL-2 and IL-12p70) when culture $\mathrm{d}$ (cultured) in basal conditions between CD and non-CD patients (data not shown), we next investigated whether the higher CD-specific response to IL-15 could occur not only as a consequence of a higher expression of its receptor, but also due to a higher basal expression of the precursors of these mediators (mRNA). Thus, mRNA expression levels of IL-15 responding molecules (iNOS, IFN $\gamma$ 
(a)

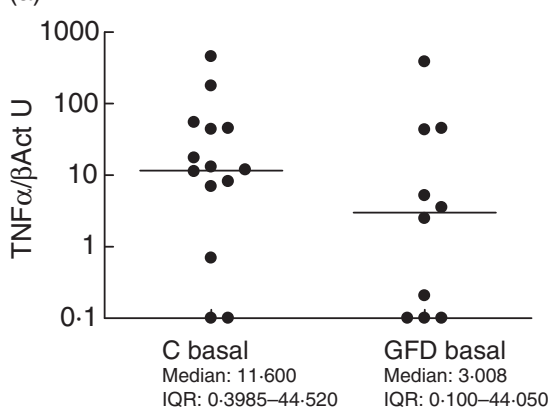

(b)

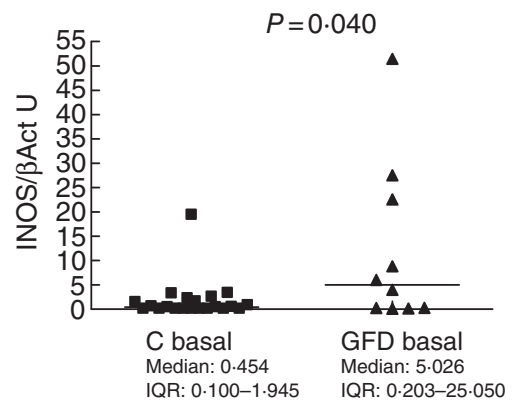

(c)

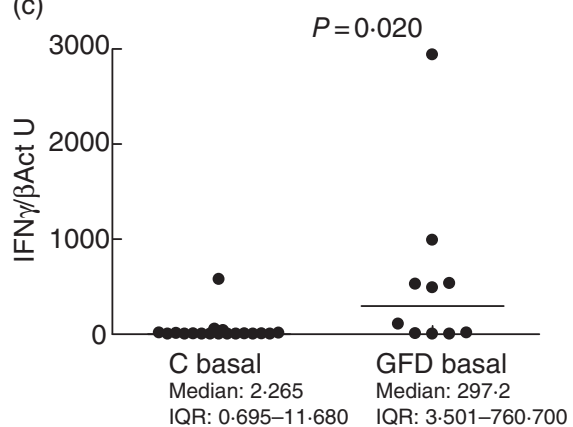

Fig. 5. Organ culture of duodenal biopsy samples: in vitro expression of TNF $\alpha$ (a), iNOS (b) and IFN $\gamma$ (c) mRNA in arbitrary units (U) in biopsy explants from non-CD healthy controls $(\mathrm{C}, n=23)$ and treated coeliac disease patients (GFD, $n=10)$ after $24 \mathrm{~h}$ of basal culture. Statistically significant differences are shown $(P<0 \cdot 05$, two-tailed Mann-Whitney $U$-test $)$. Horizontal bars indicate median values. IQR: interquartile range.

and TNF $\alpha$ ) were measured in 10 GFD-CD and 24 non-CD patients after culture in basal conditions. TNF $\alpha$ mRNA expression was similar in both groups (non-CD patients median, 11.600 U; CD patients median, 3.008; Fig. 5a). However, the basal expression of both iNOS (non-CD patients median, 0.454 U; CD patients median, 5.026; $P=0.040$ Fig. 5b) and IFN $\gamma$ (non-CD patients median, 2.265 U; CD patients median, 297.2; $P=0.020$; Fig. 5c) was found to be higher in $\mathrm{CD}$ patients.

\section{Discussion}

In this paper we report for the first time, to our knowledge, that CD patients on GFD respond more efficiently to IL-15 stimulation than non-CD individuals. We have also confirmed a higher expression of IL15R $\alpha$ in the duodenum of CD patients, as previously reported by Di Sabatino et al. [11]. The number of cells expressing IL15R was strikingly higher in CD patients on a gluten-containing diet, as compared with controls (Fig. 2). As location and morphology of positive cells were different, it also seemed clear that distinct cell lineages may express IL15R $\alpha$ in the intestinal mucosa. Interestingly, most of the IL15R $\alpha$ expression was mainly found to be associated with the nuclear membrane, as previously reported [28]. Thus, our data point to a higher expression of IL-15 binding receptor in the CD duodenum, and as only biologically active isoforms co-localize with the nuclear membrane [28], this may lead to a higher response to IL-15 in CD patients. As a consequence, and according to the two-signal model of CD immunopathogenesis [7], we propose that the first IL-15-related signal induced by gliadin would trigger more easily the development of the disease in CD patients.

Although previous studies have suggested that IL-15 remains increased in the mucosa of CD patients on GFD $[14,29]$, our results have revealed that the higher expression of its receptor is independent of IL15, because it was almost absent in CD patients on GFD. These results confirm previous observations from our group suggesting that IL15 may have an early effect in triggering the innate response, as assessed by both immunohistochemistry and Western blot [30]. Considering that this higher expression of IL15R $\alpha$ is independent of IL-15 or the inflammatory condition of the intestine, our data point to the existence of primary differences and, probably, to a new possible genetic factor involved in the CD immunopathogenesis.

Interestingly, the precursors of the effector molecules only triggered in CD (i.e. nitrites and IFN $\gamma$ ) were found to be also increased in CD patients on GFD. Although these data could question the real 'remission state' of the biopsies, all patients were reported as Marsh 0-1 at the time of the biopsy, and had negative serology for at least 1 year, therefore pointing out to a completely recovered mucosa. Moreover, TNF $\alpha$, which is induced in both groups, shows no differences in expression. It has been recently proposed that intraepithelial lymphocytes are also an important source of IFN $\gamma[31,32]$, and its production persists even after the introduction of the GFD [32]. Moreover, IFN $\gamma$ can also be an inducer of iNOS [33], and, therefore, this may explain the finding of an increased expression of both precursors in treated CD samples. As a consequence, the increased basal expression of both IL15R $\alpha$, and IFN $\gamma$ and iNOS found in our samples, can all together lead to a higher response to IL-15, resulting in a decreased immunological threshold response and the induction of the secondary pro-inflammatory signal. Therefore the higher expression of IL15R $\alpha$ would be another predisposing factor in the development of CD. It is noteworthy that most of the CD samples, both from treated and untreated patients, expressed IL15R $\alpha$ (85.71\% of treated, $78.91 \%$ of CD patients on a gluten-containing diet), while only $45 \cdot 45 \%$ of the non-CD samples had detectable levels of the precursor (Fig. 1). Moreover, the IL15R $\alpha$ expression in the CD patients did not correlate with the degree of histological lesion in untreated patients or the time on a GFD in treated patients, so its higher expression seems to be constitutive. As a consequence, IL15R $\alpha$ expression is revealed as one of the minoreffect predisposing factors involved in the CD pathogenesis (like increased tight-junction permeability, mucosal IL-15 
hypersecretion, etc.) acting all in conjunction with the main HLA-DQ2/DQ8 factor.

Although innate immunity plays a key role in the development of $\mathrm{CD}$, no specific innate factors have been described so far (TG2 and HLA-DQ2/DQ8 elicit their function in the secondary adaptive response). Moreover, there is a growing evidence about the direct toxic effect of gliadin in several biological models. It has been reported that gliadin induces rearrangements of the cytoskeleton, disassembling the integrity of the tight-junction system through a zonulindependent manner, not only in epithelial cell lines like Caco- 2 cells $[34,35]$, IEC- 6 cells $[13,35]$ or the LoVo multicelullar system [36], but also in non-coeliac biopsies challenged in vitro with gliadin [35]. Gliadin is also a potent stimulus for antigen presenting cells like monocytes, macrophages and dendritic cells, in both humans and mice [37-40]. Moreover, gliadin exerts a direct cytotoxic effect in several human cell lines by inhibiting cell growth and reducing cell viability $[37,41-43]$ and, in Caco-2 cells, gliadin inhibits DNA and RNA synthesis $[44,45]$, and induces apoptosis $[46,47]$. These results, suggesting a generalized innatetriggering effect of gliadin by a still unknown innate-gliadin receptor, led us to study the existence of an innate IL-15 response to gliadin in both $\mathrm{CD}$ and non-CD patients by using a biopsy culture model [20].

However, this model does not seem to reflect the in vivo situation, where the gliadin-induced IL-15 should be expected to have some biological effects in non-CD individuals (thought not enough for triggering the adaptive response), as suggested by the finding of IL-15 not only in the whole protein biopsy explants but even in culture supernatants after gliadin challenge, which excludes an intracellular storage of the cytokine. Therefore, after a normal gluten-containing meal, some IL-15-driven effects should be expected in all individuals (i.e. dendritic cell activation, intraepithelial lymphocyte NK-like reprogramming, increased tight-junction-mediated permeability, or enterocyte apoptosis through NKG2D-MICA interaction). Nevertheless, gliadin seems to be well tolerated by the majority of the general population and the features mentioned above are specific for CD patients. In consequence, the induced production of IL-15 might be not as biologically active in non-CD patients as observed in CD patients.

The results shown here, the increased expression of both IL15R $\alpha$ and pro-inflammatory mediators, and the higher response to IL-15, support the hypothesis that CD patients have a lower IL-15 threshold response. This hypothesis may explain why the (innate) IL-15-mediated response to gluten is not restricted to $\mathrm{CD}$, though the secondary inflammatory response is only observed in genetically susceptible CD patients. This lower threshold is probably elicited by the higher density of IL-15R $\alpha$, amongst other predisposing factors. If we consider that gliadin-induced IL-15 is peaktriggered following meals, and therefore is not a constant stimulus, we can understand how IL-15 would induce the expression of its receptor in non-CD individuals (Fig. 4a). On the other hand, in CD patients the gliadin-triggered IL-15 would not be used to induce its expression, which remains already increased, and might therefore be directly active. Moreover, it has been recently observed, by using a mice model [48], that IL15R $\alpha$ positive cells can capture and stably store IL-15 molecules as intracellular reservoirs through recycling, which then provide an environment with a prolonged release of biologically active IL-15. Therefore, the higher IL15R $\alpha$ expression in CD may act as a persistent IL-15 reservoir for the surrounding microenvironment and thus magnify its effects in the CD intestine.

In conclusion, we suggest a model in which gliadin triggers an IL-15 innate response in all individuals [20]. To explain why the IL-15 response is not so efficient in non-CD individuals, or at least in those genetically predisposed patients (HLA-DQ2/DQ8), we propose the existence of a lower IL-15 immunological threshold in CD patients for the triggering of the inflammatory response. This lower threshold would probably be mediated by the higher IL15R $\alpha$ expression, amongst other not yet determined factors, like a higher basal expression of other immune mediators. This situation renders the intestine of CD patients more susceptible to IL-15 and to the development of the secondary inflammatory response, whereas in non-CD individuals, the gliadin-triggered IL-15 would not reach the threshold level necessary to elicit the secondary immune response.

\section{Acknowledgements}

This work has been partially funded by the Spanish Ministry of Education (FPU, AP2002-2696), Spanish Ministry of Health (PI070244; 02/3068), Junta de Castilla y Leon (VA089/06, SAN196-VA17/07) and Asociación de Celiacos de Madrid (ACM).

\section{References}

1 Maki M, Collin P. Coeliac disease. Lancet 1997; 349:1755-9.

2 Sollid LM. Coeliac disease: dissecting a complex inflammatory disorder. Nat Rev Immunol 2002; 2:647-55.

3 Shan L, Molberg O, Parrot I et al. Structural basis for gluten intolerance in celiac sprue. Science 2002; 297:2275-9.

4 van de Wal Y, Kooy Y, van Veelen P et al. Selective deamidation by tissue transglutaminase strongly enhances gliadin-specific $\mathrm{T}$ cell reactivity. J Immunol 1998; 161:1585-8.

5 Qiao SW, Bergseng E, Molberg O et al. Antigen presentation to celiac lesion-derived $\mathrm{T}$ cells of a 33-mer gliadin peptide naturally formed by gastrointestinal digestion. J Immunol 2004; 173:175762.

6 Raki M, Tollefsen S, Molberg O, Lundin KE, Sollid LM, Jahnsen FL. A unique dendritic cell subset accumulates in the celiac lesion and efficiently activates gluten-reactive T cells. Gastroenterology 2006; 131:428-38.

7 Brandtzaeg P. The changing immunological paradigm in coeliac disease. Immunol Lett 2006; 105:127-39. 
8 Londei M, Ciacci C, Ricciardelli I, Vacca L, Quaratino S, Maiuri L. Gliadin as a stimulator of innate responses in celiac disease. Mol Immunol 2005; 42:913-18.

9 Jabri B, Sollid LM. Mechanisms of disease: immunopathogenesis of celiac disease. Nat Clin Pract Gastroenterol Hepatol 2006; 3:51625.

10 Maiuri L, Ciacci C, Ricciardelli I et al. Association between innate response to gliadin and activation of pathogenic $\mathrm{T}$ cells in coeliac disease. Lancet 2003; 362:30-7.

11 Di Sabatino A, Ciccocioppo R, Cupelli F et al. Epithelium derived interleukin 15 regulates intraepithelial lymphocyte Th1 cytokine production, cytotoxicity, and survival in coeliac disease. Gut 2006; 55:469-77.

12 Matysiak-Budnik T, Candalh C, Dugave C et al. Alterations of the intestinal transport and processing of gliadin peptides in celiac disease. Gastroenterology 2003; 125:696-707.

13 Clemente MG, De Virgiliis S, Kang JS et al. Early effects of gliadin on enterocyte intracellular signalling involved in intestinal barrier function. Gut 2003; 52:218-23.

14 Maiuri L, Ciacci C, Auricchio S, Brown V, Quaratino S, Londei M. Interleukin 15 mediates epithelial changes in celiac disease. Gastroenterology 2000; 119:996-1006.

15 Meresse B, Chen Z, Ciszewski C et al. Coordinated induction by IL15 of a TCR-independent NKG2D signaling pathway converts CTL into lymphokine-activated killer cells in celiac disease. Immunity 2004; 21:357-66.

16 Hue S, Mention JJ, Monteiro RC et al. A direct role for NKG2D/ MICA interaction in villous atrophy during celiac disease. Immunity 2004; 21:367-77.

17 Ebert EC. IL-15 converts human intestinal intraepithelial lymphocytes to CD94 producers of IFN-gamma and IL-10, the latter promoting Fas ligand-mediated cytotoxicity. Immunology 2005; 115:118-26.

18 Nilsen EM, Lundin KE, Krajci P et al. T cells from coeliac mucosa produce cytokines with Th1 or Th0 profile dominated by interferon gamma. Gut 1995; 37:766-76.

19 Rossi M, Young JW. Human dendritic cells: potent antigenpresenting cells at the crossroads of innate and adaptive immunity. J Immunol 2005; 175:1373-81.

20 Bernardo D, Garrote JA, Fernandez-Salazar L, Riestra S, Arranz E. Is gliadin really safe for non-coeliac individuals? Production of interleukin 15 in biopsy culture from non-coeliac individuals challenged with gliadin peptides. Gut 2007; 56:889-90.

21 Fais S, Maiuri L, Pallone F et al. Gliadin induced changes in the expression of MHC-class II antigens by human small intestinal epithelium. Organ culture studies with coeliac disease mucosa. Gut 1992; 33:472-5.

22 Budagian V, Bulanova E, Paus R, Bulfone-Paus S. IL-15/IL-15 receptor biology: a guided tour through an expanding universe. Cytokine Growth Factor Rev 2006; 17:259-80.

23 Bongers M, Liehl E, Barsig J. One-step RT-PCR to detect cytokine/ chemokine induction in macrophages. Focus 2003; 21:66-8.

24 Tejman-Yarden N, Zlotnik M, Lewis E, Etzion O, Chaimovitz C, Douvdevani A. Renal cells express a functional interleukin-15 receptor. Nephrol Dial Transplant 2005; 20:516-23.

25 Ohtsuki M, Nomura T, Morimoto S et al. Suppressed expression of GTP cyclohydrolase I mRNA and accelerated expression of inducible nitric oxide synthase mRNA in endomyocardial biopsy specimens from patients with dilated cardiomyopathy. Clin Chim Acta $2005 ; 353: 103-7$.
26 Karlsson MG, Ludvigsson J. Determination of mRNA expression for IFN-gamma and IL-4 in lymphocytes from children with IDDM by RT-PCR technique. Diabetes Res Clin Pract 1998; 40:2130.

27 Atkins GJ, Haynes DR, Geary SM, Loric M, Crotti TN, Findlay DM. Coordinated cytokine expression by stromal and hematopoietic cells during human osteoclast formation. Bone 2000; 26:653-61.

28 Dubois S, Magrangeas F, Lehours P et al. Natural splicing of exon 2 of human interleukin-15 receptor alpha-chain mRNA results in a shortened form with a distinct pattern of expression. J Biol Chem 1999; 274:26978-84.

29 Mention JJ, Ben Ahmed M, Begue B et al. Interleukin 15: a key to disrupted intraepithelial lymphocyte homeostasis and lymphomagenesis in celiac disease. Gastroenterology 2003; 125:730-45.

30 Leon AJ, Garrote JA, Blanco-Quiros A et al. Interleukin 18 maintains a long-standing inflammation in coeliac disease patients. Clin Exp Immunol 2006; 146:479-85.

31 Olaussen RW, Johansen FE, Lundin KE, Jahnsen J, Brandtzaeg P, Farstad IN. Interferon-gamma-secreting $\mathrm{T}$ cells localize to the epithelium in coeliac disease. Scand J Immunol 2002; 56:65264.

32 Forsberg G, Hernell O, Melgar S, Israelsson A, Hammarstrom S, Hammarstrom ML. Paradoxical coexpression of proinflammatory and down-regulatory cytokines in intestinal $\mathrm{T}$ cells in childhood celiac disease. Gastroenterology 2002; 123:667-78.

33 Maiuri MC, De Stefano D, Mele G et al. Gliadin increases iNOS gene expression in interferon-gamma-stimulated RAW 264.7 cells through a mechanism involving NF-kappa B. Naunyn Schmiedebergs Arch Pharmacol 2003; 368:63-71.

34 Sander GR, Cummins AG, Henshall T, Powell BC. Rapid disruption of intestinal barrier function by gliadin involves altered expression of apical junctional proteins. FEBS Lett 2005; 579:4851-5.

35 Drago S, El Asmar R, Di Pierro M et al. Gliadin, zonulin and gut permeability: effects on celiac and non-celiac intestinal mucosa and intestinal cell lines. Scand J Gastroenterol 2006; 41:408-19.

36 Jelinkova L, Tuckova L, Cinova J, Flegelova Z, Tlaskalova-Hogenova H. Gliadin stimulates human monocytes to production of IL-8 and TNF-alpha through a mechanism involving NF-kappaB. FEBS Lett 2004; 571:81-5.

37 Dolfini E, Elli L, Roncoroni L et al. Damaging effects of gliadin on three-dimensional cell culture model. World J Gastroenterol 2005; 11:5973-7.

38 Nikulina M, Habich C, Flohe SB, Scott FW, Kolb H. Wheat gluten causes dendritic cell maturation and chemokine secretion. J Immunol 2004; 173:1925-33.

39 Thomas KE, Sapone A, Fasano A, Vogel SN. Gliadin stimulation of murine macrophage inflammatory gene expression and intestinal permeability are MyD88-dependent: role of the innate immune response in Celiac disease. J Immunol 2006; 176:2512-21.

40 Cinova J, Palova-Jelinkova L, Smythies LE et al. Gliadin peptides activate blood monocytes from patients with celiac disease. J Clin Immunol 2007; 27:201-9.

41 Hudson DA, Cornell HJ, Purdham DR, Rolles CJ. Non-specific cytotoxicity of wheat gliadin components towards cultured human cells. Lancet 1976; 1:339-41.

42 Rocca E, Paganuzzi Stammati A, Zampaglioni F, Zucco F. Effects of gliadin-derived peptides from bread and durum wheats on in vitro cultures of human cell lines. Implications for coeliac disease pathogenesis. Toxicol Lett 1983; 16:331-8.

43 Dolfini E, Elli L, Dasdia T et al. In vitro cytotoxic effect of bread 
wheat gliadin on the LoVo human adenocarcinoma cell line. Toxicol In Vitro 2002; 16:331-7.

44 Giovannini C, Mancini E, De Vincenzi M. Inhibition of the cellular metabolism of Caco- 2 cells by prolamin peptides from cereals toxic for coeliacs. Toxicol In Vitro 1996; 533-8.

45 Giovannini C, Lechetti R, De Vincenzi M. The activities of peptides ' 3143 ', '44-55' and '56-68' of A-gliadin on in vitro cultures of CaCo-2 cells. ATLA 1997; 25:437-43.

46 Giovannini C, Sanchez M, Straface E, Scazzocchio B, Silano M, De
Vincenzi M. Induction of apoptosis in caco- 2 cells by wheat gliadin peptides. Toxicology 2000; 145:63-71.

47 Giovannini C, Matarrese P, Scazzocchio B et al. Wheat gliadin induces apoptosis of intestinal cells via an autocrine mechanism involving Fas-Fas ligand pathway. FEBS Lett 2003; 540:117-24.

48 Sato N, Patel HJ, Waldmann TA, Tagaya Y. The IL-15/IL-15Ralpha on cell surfaces enables sustained IL-15 activity and contributes to the long survival of CD8 memory T cells. Proc Natl Acad Sci USA 2007; 104:588-93. 